\title{
DETERMINE TASK DEMAND FROM BRAIN ACTIVITY
}

\author{
Matthias Honal and Tanja Schultz \\ Carnegie Mellon University, 407 South Craig Street, Pittsburgh 15213, PA, USA \\ Karlsruhe University, Am Fasanengarten 5, 78131 Karlsruhe, Germany \\ honal@ira.uka.de,tanja@\{ira.uka.de,cs.cmu.edu\}
}

Keywords: Human-centered systems, Brain Activity, EEG, Task Demand Identification, Meeting and Lecture Scenario.

Abstract: Our society demands ubiquitous mobile devices that offer seamless interaction with everybody, everything, everywhere, at any given time. However, the effectiveness of these devices is limited due to their lack of situational awareness and sense for the users' needs. To overcome this problem we develop intelligent transparent human-centered systems that sense, analyze, and interpret the user's needs. We implemented learning approaches that derive the current task demand from the user's brain activity by measuring the electroencephalogram. Using Support Vector Machines we can discriminate high versus low task demand with an accuracy of $92.2 \%$ in session dependent experiments, $87.1 \%$ in session independent experiments, and $80.0 \%$ in subject independent experiments. To make brain activity measurements less cumbersome, we built a comfortable headband with which we achieve $69 \%$ classification accuracy on the same task.

\section{INTRODUCTION}

Our modern information society increasingly demands ubiquitous mobile computing systems that offer its users seamless interaction with everybody, everything, everywhere, at any time. Although the number and accessibility of mobile devices such as laptop computers, cell phones, and personal digital assistants grows rapidly, the effectiveness in supporting the users to fulfilling their tasks proves to be much smaller than expected. This mainly results from the fact that such devices lack situational awareness and sense for the users' needs. As a consequence users waste their time with manually configuring inflexible devices rather than obtaining relevant information and efficient automatic support to solve their problems and tasks at hand.

It is our believe that the solution lies in intelligent transparent human-centered systems that sense, analyze, and interpret the needs of their users, then adapt themselves accordingly, provide the optimal support to given problems, and finally present the relevant results in an appropriate way. The goal of the work presented here is to solve the analytical part of human-centered systems, i.e. sensing, analyzing, and interpreting the users' needs.

For this purpose we develop learning approaches that derive the users' condition from their brain activity. We are interested in conditions that are important in the context of human-computer interaction and human-human communication. In this particular study we focus on the (mental) task demand as a user condition in the context of lecture presentations and meetings.

The term task demand defines the amount of mental resources required to execute a current activity. Although we are using the general term task demand, we are exclusively concerned about the mental not the physical task demand. Task demand information can be helpful in various situations, e.g. while driving a car, operating machines, or performing other critical tasks. Depending on the level of demand and cognitive load, any distraction arising from electronic devices such as text messages, incoming phone calls, traffic or navigation information, etc. should be suppressed or delayed. Also, the analysis of task demand during computer interaction allows to asses usability. In a lecture scenario, a speaker may use task demand information to tailor the presentation toward the audience.

In this paper we investigate the potential of detecting task demand by measuring the brain activity using scalp electrodes. Although we focus on the system evaluation in the lecture and meeting scenario, the described methods are applicable to any other reallife situation. To make electrode-based recordings acceptable, the following issues must be addressed: 
- Robustness: The system needs to be robust against artefacts introduced by speech or body movement

- Usability: EEG sensors and recording device need to be user friendly and comfortable to wear

- Applicability: Measuring brain activity must be feasible in realistic scenarios in real-time.

In this work we are addressing these three goals by relaxing the inconveniences of clinical brain activity recording and make it applicable to real humancomputer interaction and human-human communication scenarios.

\section{ELECTROENCEPHALOGRAM}

The source of the Electroencephalogram (EEG) is neural activity in the cortex, the outmost part of the human brain. This neural activity causes electrical potential differences, which can be measured using scalp electrodes. Information between neurons is transferred via the synapses where chemical reactions take place causing ion movements. These movements result in excitatory or inhibitory electrical potentials in the post-synaptic neurons. The electrical fields emerging from the ion movements are called cortical field potentials and have a dipole structure. If the electrical activity of a huge number of neurons is synchronized, the corresponding dipoles point all in the same direction. Their sum becomes large enough such that potential differences between particular scalp positions and a constant reference point can be measured. EEG characteristics like frequency, amplitude, temporal and topographic relations of certain patterns can then be used to make inferences about underlying neural activities (Zschocke, 1995).

In the EEG which can be measured at the scalp, amplitudes between $1 \mu \mathrm{V}$ and $100 \mu \mathrm{V}$ and frequencies between $0 \mathrm{~Hz}$ and $80 \mathrm{~Hz}$ can be observed. These EEG signals show specific characteristics at different scalp positions, depending on the current mental condition. When the human brain is not absorbed by external sensory stimuli or other mental processes, we usually observe the $\alpha$-activity across the cortex, i.e. rhythmic signals between $8 \mathrm{~Hz}$ and $13 \mathrm{~Hz}$ with large amplitudes. When performing higher mental processes the $\alpha$-activity is attenuated and other activity patterns occur in those cortex regions, where the processes happen. In many cases these patterns are identified by $\gamma$-activity, which typically show frequencies around $40 \mathrm{~Hz}$ and have a lower amplitude than $\alpha$-activity (Schmidt and Thews, 1997). In this work we assume that the degree of $\alpha$-activity attenuation and activity at higher frequencies is correlated with task demand. This is justified by the fact that the amplitude of non- $\alpha$ activity is correlated with the degree of vigilance, a physiological continuum between sleepiness and active alertness (Zschocke, 1995). Furthermore, it is known that people are more alert when the task demand is high. The frequency analysis of our recorded data confirms this assumption. During most activity types several cortex regions are involved and task demand is characterized by the amplitude of non- $\alpha$-activity in all regions involved in the current task. This suggests that the activity of the whole cortex must be taken into account to achieve optimal results for task demand estimation.

\section{TASK DEMAND \& VIGILANCE}

A large body of research work concerns the computational analysis of brain activity, applying EEG, functional magnetic resonance imaging, and functional near infrared spectroscopy to areas such as estimation of mental task demand. Several groups reported research on the computational assessment of task demand based on EEG data recorded while varying the task difficulty (Smith, 2001), (PleydellPearce, 2003), (Berka, 2004). These studies focused on the design of intelligent user interfaces that optimize operator performance by adjusting to the predicted task demand level. Regression models were trained to predict task demand from the recorded EEG data. These models used the task difficulty or the rate of errors as references during task execution. The features extracted from the EEG data represented mostly the frequency content of the signals. Positive correlations between predictions and references or predictions and self-estimates of task demand (Smith, 2001) are reported throughout these studies. Pleydell-Pearce (2003) achieved a classification accuracy of $72 \%$ for the discrimination of low versus high task demand in subject and session dependent experiments and $71 \%$ in subject independent experiments. Task demand assessment has also been done on data from other modalities, including muscular activity (Pleydell-Pearce, 2003), blood hemodynamics (Izzetoglu, 2004), and pupil diameter (Iqbal, 2004). Reasonable results could be achieved with all three modalities. However, correlations between pupil diameter and task demand could only be shown for one interactive task out of a group of various cognitive tasks.

Other work focused on the EEG-based estimation of operator's vigilance during sustained attention tasks (e.g. car driving or operating a power plant). Jung (1997) asked subjects to respond to auditory stimuli which simulate sonar target detection, while EEG was recorded from five 
electrodes over the parietal, central and occipital cortex. The error rate in terms of failures to respond to stimuli was then used as reference for a MultiLayer ANN which was trained with a frequency representation of the EEG signals to predict a vigilance index between 0 and 1 . On unknown data a root mean square error (RMS-error) of 0.156 between predictions and references is reported for a subject dependent experimental setup. Duta et al. (Duta, 2004) recorded EEG from the mastoids while subjects had to perform visual attention tasks. Vigilance was labelled by experts who visually inspected the recorded data. Three vigilance categories "alertness", "intermediate" and "drowsiness" were distinguished. Using the coefficients of an AR model as features for Multi-Layer ANNs $39 \%$ to $62 \%$ predictions matched the references in subject independent experiments.

\section{DATA \& METHODS}

\subsection{Data Capturing}

Two different devices were used for data acquisition: an EEG-cap from ElectroCap ${ }^{\mathrm{TM}}$ and a self-made EEG-headband (see Figure 1). The majority of data were recorded with the ElectroCap ${ }^{\mathrm{TM}}$ using 16 electrodes placed at positions $\mathrm{fp} 1, \mathrm{fp} 2, \mathrm{f} 3$, $\mathrm{f} 4, \mathrm{f} 7, \mathrm{f8}, \mathrm{fz}, \mathrm{t} 3, \mathrm{t} 4, \mathrm{t} 5, \mathrm{t} 6, \mathrm{p} 3, \mathrm{p} 4, \mathrm{pz}, \mathrm{o} 1$, and o2 according to the international 10-20 system (Jasper, 1958). Reference electrodes were attached to the ear lobes and linked together before amplification. Although we are aware of the relationship between facial expressions and level of task demand, we decided to exclude the motor cortex from our measurement for two reasons: firstly, the facial muscular activity is partly captured by the frontal EEG electrodes, and secondly we assume that motor activity is of rather minor importance for the assessment of our classification task.

Some data were recorded with a headband, in which we sewed in four electrodes at the forehead positions $\mathrm{fp} 1, \mathrm{fp} 2, \mathrm{f} 7$, and $\mathrm{f} 8$. Reference electrodes were attached to the mastoids and linked together before amplification, the ground electrode was placed at the back of the neck. The headband has three major advantages over the ElectroCap ${ }^{\mathrm{TM}}$ which are crucial to real-life applications: the headband is (1) more comfortable to wear, (2) much easier to attach, and (3) better to maintain and clean, also no electrode gel gets in contact with the subject's hair. The drawback is the limited positioning and number of electrodes compared to the ElectroCap ${ }^{\mathrm{TM}}$.

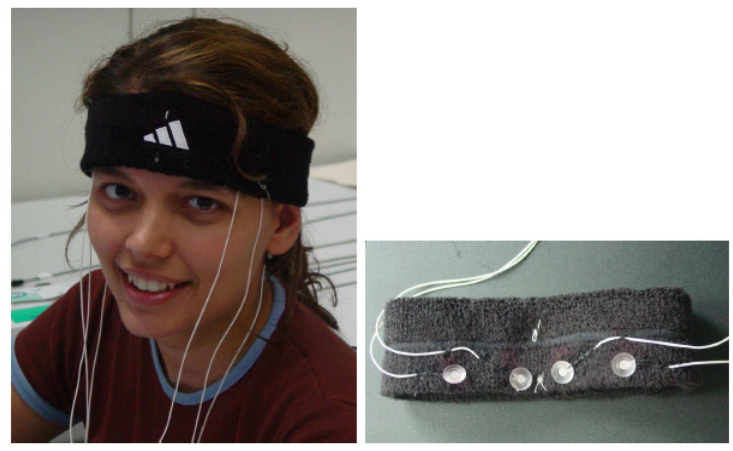

Figure 1: Headband, build-in electrodes at fp1, fp2, f7, f8.

In contrast to recordings for clinical purposes, subjects were allowed to move freely during the recordings to keep the situation as natural as possible, i.e. the subject's head was not fixated. Consequently we had to deal with data artefacts introduced by muscular activity (some recordings required speaking as well). Strategies to remove those artefacts will be described in section 4.2.

Amplification and A/D-conversion was done with a 16 channel VarioPort ${ }^{\mathrm{TM}}$ physiological data recorder (Becker, 2005). Each channel had an amplification factor of 2775 and a frequency range from $0.9 \mathrm{~Hz}$ to $60 \mathrm{~Hz}$. After amplification, A/D conversion was performed using 4096 A/D-steps and a sampling rate of $256 \mathrm{~Hz}$. The data was transferred instantaneously from the amplifier to a computer via an RS232 port for online processing. The port capacity is limited to 115200 Bits per second which corresponds to 28 electrode channels at a sampling of $256 \mathrm{~Hz}$. Although sampling with a lower frequency should be sufficient to avoid aliasing when considering the amplifier's upper cutoff-frequency of $60 \mathrm{~Hz}$, we decided to go with $256 \mathrm{~Hz}$ since for technical reasons the slope of the band pass filter is very small.

\subsection{Data Preprocessing}

Figure 2 summarizes the signal processing steps of our task demand estimation system. After EEG recording, artefacts are removed applying independent component analysis (ICA). A short time Fourier transform (STFT) is used for feature extraction. After feature normalization and averaging over temporally adjacent features, different methods for reducing the dimensionality are used. Finally, Support Vector Machines (SVMs) or Artificial Neural Networks (ANNs) for classification or regression are applied to obtain task demand predictions. We also applied SelfOrganizing-Maps (SOMs) to determine which levels of task demand can be reliably discriminated. 


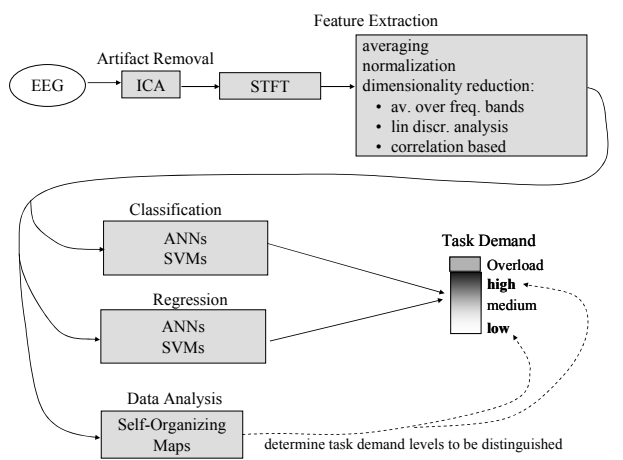

Figure 2: Task Demand Estimation System.

\subsubsection{Artefact Removal}

Artefacts such as muscular activity and especially eye movements contaminate the EEG signal, since the corresponding electrical potentials are an order of magnitude larger than the EEG sources. This causes in particular problems in the EEG that is measured over the frontal cortex. ICA has shown to be very efficient for artefact removal in EEG data (Jung et al., 2000).

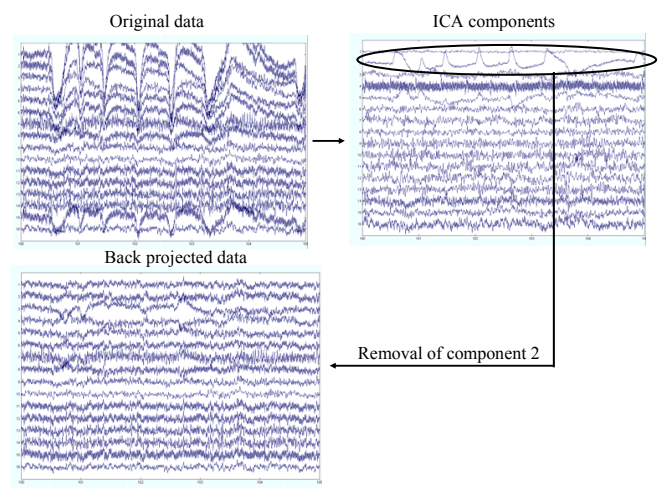

Figure 3: Artefact removal applying ICA: (1) independent components are computed from the original data (top left), (2) the second component (eye blinking artefact) is identified and rejected (top right), and (3) the data is projected back to the original space (bottom left).

To apply ICA to EEG data it is assumed that the signal measured at one electrode can be described by a linear combination of signals emerging from independent processes (i.e. cortical field potentials, muscular artefacts, $60 \mathrm{~Hz} \mathrm{AC}$ noise $)$ : Let $\mathbf{x}(\mathrm{t})$ be the vector of signals measured at all electrodes at time $t$ and $\mathbf{s}(\mathrm{t})$ be the independent components. Then $\mathbf{x}(\mathrm{t})$ can be expressed by $\mathbf{x}(\mathrm{t})=\mathrm{A} \cdot \mathbf{s}(\mathrm{t})$, where A is called mixing matrix. ICA computes the matrix $A$, or its inverse the de-mixing matrix $\mathrm{W}$, such that independent components can be estimated from the measured signals (Hyväarinen et al., 2000). Artefact components can then be identified either by visual inspection of the training data or by using crossvalidation and be rejected from the data. The remaining components are projected back into the original coordinate system (see Figure 3). For ICA computation we used the open source Matlab toolbox EEGLAB (Delorme et al., 2004), which applies the Informax algorithm to the matrix estimation.

\subsubsection{Feature Extraction, Averaging and Normalization}

After artefact removal we computed the power spectrum of the time signal applying STFT. For twosecond long segments overlapping by one second, features were computed representing the content of frequency bands with $0.5 \mathrm{~Hz}$ width. This results in one feature vector per second. The dimensionality of one feature vector for 16 electrode channels and frequencies ranging from 0 to $45 \mathrm{~Hz}$ is $16 \cdot 90=1440$. To reduce the influence of outliers final feature vectors for each time point were obtained by averaging over $k$ previous features. To compensate for different ranges in the frequency bands, we normalized each feature using the following two normalization approaches:

- GlobalNorm: Feature means and variances are calculated based on the complete training set. Calculated values are used globally for mean subtraction and variance normalization on all data (training, validation, and test data).

- UserNorm: Feature means and variances are calculated on training, validation, and test data separately for each user. Then, user-specific mean subtraction and variance normalization is applied.

\subsubsection{Feature Reduction}

Since the dimensionality of the feature vector may be large compared to the amount of training data, we investigated various feature reduction methods. A straightforward approach is to average over adjacent frequency bands, another approach is the Linear Discriminant Analysis (LDA), which selects features according to their discriminative power (Fukunaga, 1972). For sparse data and large dimensionalities, LDA estimation may become ill-conditioned. Therefore, we also applied a correlation-based feature reduction method, which selects those features that correlate best with the variable to be predicted. This method proved to be particular useful for the assessment of task demand, since - in contrast to LDA - it takes into account the continuous nature of the predicted variable. 


\subsection{Data Analysis}

To learn more about the data structure and to gain insights into the granularity and distinctness of task demand levels, we generated self-organizing maps (SOMs) (Kohonen, 1995) for the training data. After obtaining the Best Matching Unit (BMU) for each training example, a map was calculated which visualizes colour-coded clusters corresponding to different task demand levels. Thus the spatial relation between the feature vectors belonging to the different task demand levels can be visualized concisely on a two dimensional grid. Although the SOM-based analysis may indicate which task demand levels are easy to discriminate, the hypotheses have to be verified experimentally on test data. SOM training and visualization were performed with the MATLAB ${ }^{\text {TM }}$ based SOMToolbox (Vesanto et al., 2000).

\subsection{Learning Methods}

We investigated two types of classifiers: Multilayer ANNs and SVMs. ANN classifiers were trained with standard back-propagation, based on feed-forward networks with a tanh activation function and one hidden layer. For all ANNs early stopping regularization was performed and the number of neurons in the hidden layer was determined on the validation data. For SVM-based classification we used an implementation of $\mathrm{SVM}^{\text {light }}$ (Joachims, 1999), which directly addresses the multi-class problem (Tsochantaridis, 2004). SVMs were restricted to linear kernels to limit computational costs and avoid extensive parameter tuning. By treating the task demand levels as class labels (e.g. "low", "medium", "high"), both classification methods can be applied to the problem of task demand estimation. To exploit the information contained in the ordinal scaling of the different class labels, we investigated the regression versions of ANNs and SVMs as well.

Since ANN predictions fluctuate due to random weight initializations, predictions from five networks trained on the same data were combined using majority decisions (in case of classification) or averaging (in case of regression).

\subsection{Evaluation Methods}

The system performance for task demand assessment is evaluated in terms of classification accuracy. When regression methods are used, class labels are assigned numeric values and each prediction is assigned to the label with the closest value. Although confusion matrices could lead to a deeper understanding of pros and cons of the prediction methods, we decided to use the more concise classification accuracies. Results presented here are averages over all test sets and all class accuracies. The latter gives more reliable results in the presence of unbalanced test sets.

We use the normalized expected loss to compare accuracies that were calculated based on different numbers of classes. Comparing accuracies directly would not be appropriate since the chance accuracy $\mathrm{A}^{(\mathrm{c})}$ varies with the number of classes. The normalized expected loss relates the observed error to the chance error and thus makes it independent from the number of classes. The value of the normalized expected loss is bound by $1 / \mathrm{A}^{(\mathrm{c})}$ and ranges between 0 and 1 .

\section{EXPERIMENTS}

We conducted various experiments to evaluate task demand assessment and collected EEG data for this purpose, using both the headband and the ElectroCap $^{\mathrm{TM}}$. In offline experiments we analyzed and optimized the processing steps of the system.

\subsection{Data Collection}

Task demand data was collected from subjects perceiving an audio-visual slide presentation. The presentations were tailored to the subjects' educational background and designed to provoke each task demand level with equal amount of time. The presentations were video-taped so that each of the subjects could evaluate their task demand afterwards by watching the tape. We defined the following task demand levels:

- Low: All details of the presentation are well understood with low mental effort.

- Medium: Some mental effort is required to follow the presentation, not all details may be understood.

- High: All available mental resources are required to understand at least the essence of the topic. Most of the details are not understood.

- Overload: The presentation topic is not understood. The subject is overwhelmed, disengaged and makes no more effort to understand the presentation.

In total 7690 seconds of data were recorded with the ElectroCap ${ }^{\mathrm{TM}}$ from six students (three male, three female) between 23 and 26 years old. One subject was recorded twice. 1918 seconds of data were recorded with the headband from two students (one male, one female) between 21 and 28 years old. 


\subsection{Experimental Setup}

One major goal of our experiments was to investigate the impact of user and session dependencies on the system performance. The other goal was to examine the efficiency and performance of the headband compared to the ElectroCap ${ }^{\mathrm{TM}}$. We therefore conducted user/session dependent and independent experiments on ElectroCap ${ }^{\mathrm{TM}}$ and headband recordings using the following setup:

UD: User and session dependent setup: Different subsets of the same session were used for training $(80 \%)$, validation $(10 \%)$, and testing $(10 \%)$. Four sessions were recorded with the ElectroCap ${ }^{\mathrm{TM}}$ and two with the headband.

UI: User and session independent setup: The system was trained on three of the four ElectroCap ${ }^{\mathrm{TM}}$ recording sessions and tested on the fourth session in a round-robin fashion. For better comparability the same test sets as for setup UD were used. Validation was performed on two held-out ElectroCap $^{\mathrm{TM}}$ recording sessions.

SI: Session independent but user dependent setup: One subject was recorded twice in two separate sessions using the ElectroCap ${ }^{\mathrm{TM}}$. The system was trained on one session and tested on the other, without validation set.

\subsection{Results - Data Analysis}

Figure 4 compares for one subject the SOM trained on all task demand levels (left-hand side) to the SOM trained on high and low task demand level (right-hand side). The grey-scaled dots represent the best matching units (BMUs) on the grid belonging to the feature vectors of different task demand levels. The size of the dots is proportional to the amount of feature vectors that share the same BMU. Obviously we see a large overlap between the BMUs when all four task demand levels are considered, while the BMUs for low and high task demand seem to be well separable. Same observations were made for the SOMs trained on other subjects.

Baseline results on the UD setup (no averaging, GlobalNorm normalization, no feature reduction, linear classification SVMs) confirmed our expectation that the four task demand levels are difficult to discriminate (classification accuracy $40 \%$, normalized expected loss 0.81). When distinguishing low versus high task demand we achieved a classification accuracy of $78 \%$ and a normalized expected loss of 0.43 . The major reason for the poor results on discriminating all four levels is that subjects had difficulties to identify the boundaries between adjacent demand levels. To investigate this we asked some subjects to re- evaluate their task demand at a later time. We found a low intracoder agreement among adjacent task demand levels, while high versus low task demands were rarely confused. In the remainder of this section we will therefore focus on the discrimination between low and high task demand.

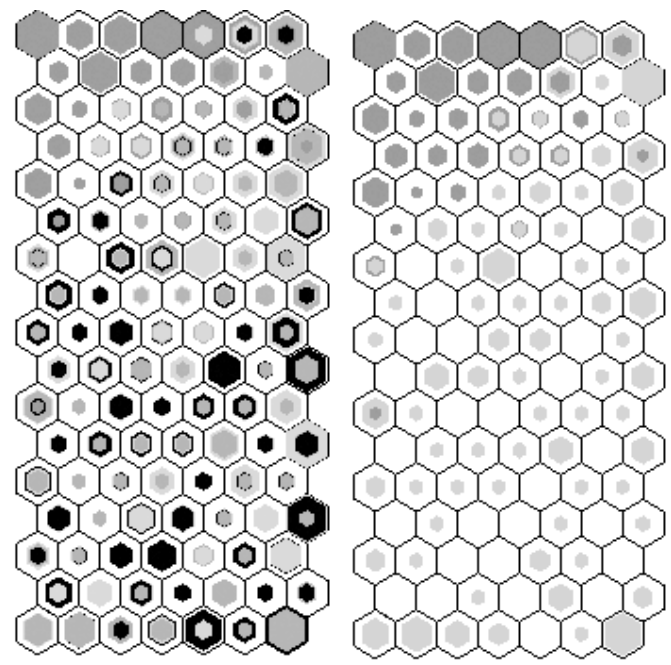

Figure 4: SOM trained on all four task demand levels (lefthand side) and on low vs. high task demand (right-hand side). Grey scale intensity indicates task demand level, ranging from low (light) to overload (dark).

Table 1 shows the average amount of data per subject after removing the medium and overload task demand recordings.

Table 1: Data per subject (in seconds) for all setups.

\begin{tabular}{|c|c|c|c|}
\hline Setup & Training & Validation & Test \\
\hline UD & 247 & 31 & 64 \\
\hline UI & 740 & 229 & 64 \\
\hline SI & 257 & - & 48 \\
\hline
\end{tabular}

\subsection{Results - Learning Method}

Table 2 compares the regression and classification versions of ANNs and SVMs for the baseline system (no averaging, GlobalNorm normalization, no feature reduction). For all three experimental setups SVMs perform better than ANNs. For setup SI the regression SVMs significantly outperform the classification SVMs. For the other setups the differences between the two SVM variants are rather small. Since at least theoretically the regression SVMs should be able to better exploit the ordinal scaled information given in the task demand levels, we decided to use these in the remainder of our experiments. 
Table 2: Baseline system performance for all setups; classification $\left({ }^{c}\right)$ and regression methods $\left({ }^{r}\right)$; In parentheses: standard deviation for five ANN experiments.

\begin{tabular}{|l|l|l|l|}
\hline Setup & UD & UI & SI \\
\hline SVM c & $\mathbf{8 1 \%}$ & $72 \%$ & $66 \%$ \\
\hline SVM r & $79 \%$ & $74 \%$ & $\mathbf{7 3 \%}$ \\
\hline ANN c & $78 \%(7 \%)$ & $70 \%(3 \%)$ & $53 \%(5 \%)$ \\
\hline ANN r & $71 \%(3 \%)$ & $69 \%(3 \%)$ & $66 \%(5 \%)$ \\
\hline
\end{tabular}

\subsection{Results - Normalization and Feature Reduction}

In the following experiments we optimized the processing steps of our system in a greedy fashion on the validation set. Table 3 shows the classification accuracies for all experimental setups with the optimal parameters (given in parentheses).

Averaging over $k=2$ feature vectors improved the results for the UD and UI setup. The use of normalization method UserNorm instead of the baseline method GlobalNorm improved results for setups UI and SI. This matches our expectation, since this method reduces the variability across sessions (UI and SI) as well as across subjects (UI). Normalization is not relevant for the user dependent setup (UD) since it only applies when data of different subjects are used for training and test.

Table 3: Results for the optimized task demand system.

\begin{tabular}{|l|l|l|l|}
\hline Setup & UD & UI & SI \\
\hline Baseline & $78 \%$ & $74 \%$ & $73 \%$ \\
\hline Averaging $(k=2)$ & $82 \%$ & $79 \%$ & $73 \%$ \\
\hline $\begin{array}{l}\text { Normalizing } \\
\text { (UserNorm) }\end{array}$ & N/A & $\mathbf{8 0 \%}$ & $\mathbf{8 7 \%}$ \\
\hline $\begin{array}{l}\text { Feature Reduction } \\
\text { (Corr-based) }\end{array}$ & $\mathbf{9 2 \%}$ & $77 \%$ & $66 \%$ \\
\hline
\end{tabular}

Feature reduction was only successful for UD, where a correlation based reduction from 1440 to 80 features yielded considerable improvements. For the other setups feature reduction did not help, probably since despite normalization the data variability was too large. Consequently, features which were well correlated with task demand on the training data exhibited poor correlations with task demand on the test data. Comparing the results of feature reduction among the different setups is difficult since the optimal number of 80 features for the UD setup was determined on the validation set, while we set this number manually to 240 for the SI and UI setup as the validation method did not give any reasonable optimum.

Averaging over adjacent frequency bands for feature reduction corresponds to putting features into bins of size $b$. We observed that even for large numbers of $b$ the results did not drop much for any of the setups. For $b=45$ (two features per electrode, i.e. lower and the upper frequencies) results are in the same range as without feature reduction. For $b=90$ (one feature per electrode, 8 features in total) results dropped significantly. This suggests that it is sufficient to consider for task demand estimation the content of two broad frequency bands: the lower frequencies (around the $\alpha$-band) and the higher frequencies (around the $\gamma$-band). Experiments to investigate this hypothesis are planned. The feature reduction would benefit from more reliable model estimation and reduced computational costs.

\subsection{ElectroCap ${ }^{\mathrm{TM}}$ versus Headband}

After optimizing the system parameters, experiments using the UD setup were conducted on the headband data. A classification accuracy of $69 \%$ could be achieved. This compares to $69 \%$ using the four ElectroCap $^{\mathrm{TM}}$ recordings with 4 electrodes and $82 \%$ with 16 electrodes. These results were achieved without correlation based feature reduction. For the reduced number of electrodes, the classification accuracies for half of the subjects are at least $86 \%$ or better, while for the other half they are around chance. This implies that the feasibility of task demand estimation based on four electrodes might depend on the subject or even on the presentation itself. As described above the presentations and topics were tailored towards the educational background of the subjects.

\section{CONCLUSIONS}

In this paper we described our efforts in building human-centered systems that sense, analyze, and interpret the users' needs. We implemented several learning approaches that derive the task demand from the user's brain activity. Our system was built and evaluated in the domain of meeting and lecture scenarios. For the prediction of low versus high task demand during a presentation we obtained accuracies of $92 \%$ in session dependent experiments, $87 \%$ in subject dependent but session independent experiments, and $80 \%$ in subject independent experiments. To make brain activity measurements less cumbersome, we built a comfortable headband with which we achieved $69 \%$ classification accuracy for low versus high task demand discrimination. Based on our findings we developed an online system that derives user states from brain activity using the headband (Honal et al., 2005). A screenshot of our prototype is shown in Figure 5. 


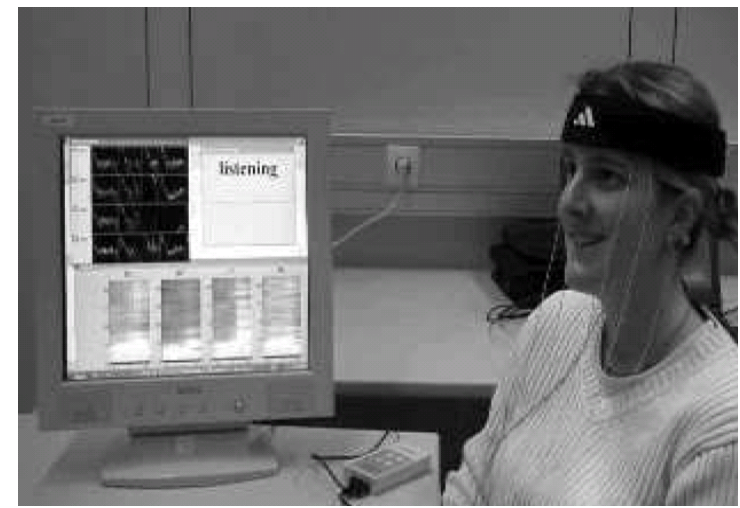

Figure 5: Screenshot of our prototype online brain activity system. The upper left monitor area displays the EEG signal; the hypothesized current user state is shown in the upper right corner. Spectrograms for the headband electrodes fp1, fp2 $\mathrm{f} 7$ and $\mathrm{f} 8$ are shown at the bottom.

\section{ACKNOWLEDGEMENTS}

This material is in part based upon work supported by the European Union (EU) under the integrated project CHIL (Grant number IST-506909). Any opinions, findings, and conclusions are those of the authors and do not necessarily reflect the views of the EU. The authors would like to thank Laura Honal and Dana Burlan for their cooperation and patience during the data collection, Christian Mayer and Markus Warga for implementing the recording software and tools, as well as Klaus Becker and Gerhard Mutz for their support with respect to the recording devices. Without the contributions of all these people, this work would not have been possible.

\section{REFERENCES}

Becker, K. VarioPort ${ }^{\mathrm{TM}}$. http://www.becker-meditec.com/. Berka, C., Levendowski, D., Cvetinovic, M., et al., 2004. Real-Time Analysis of EEG Indexes of Alertness, Cognition, and Memory Acquired With a Wireless EEG Headset. Int. Journal of HCI, 17(2):151-170.

Delorme A., Makeig, S., 2004. EEGLAB: an open source toolbox for analysis of single-trial EEG dynamics. Journal of Neuroscience Methods, 134:9-21.

Duta, M., Alford, C., Wilon, S., and Tarassenko, L., 2004. Neural Network Analysis of the Mastoid EEG for the Assessment of Vigilance. Int. Journal of HCI, 17(2):171-195.

Electro-Cap ${ }^{\mathrm{TM}}$, Electro-Cap International, Inc.: http://www.electro-cap.com/.
Fukunaga, K., 1972. "Introduction to Statistical Pattern Recognition", Academic Press, New York, London.

Honal, M. and Schultz, T., 2005. Identifying User State using Electroencephalographic Data, Proceedings of the International Conference on Multimodal Input (ICMI), Trento, Italy, October 2005.

Hyväarinen, A. and Oja, E., 2000. Independent Component Analysis: Algorithms and Applications. Neural Networks, 13(4-5):411-430.

Iqbal, S., Zheng, X., and Bailey, B., 2004. Task evoked pupillary response to mental workload in human computer interaction. In Proceedings of Conference of Human Factors in Computer Systems (CHI).

Izzetoglu, K., Bunce, S., Onaral, B., Pourrezaei, K., and Chance, B., 2004. Functional Optical Brain Imaging Using Near-Infrared During Cognitive Tasks. International Journal of HCI, 17(2):211-227.

Jasper, H. H.. 1958. The ten-twenty electrode system of the International Federation. Electroencephalographic Clinical Neurophysiolgy, 10:371-375.

Joachims, T. (1999). Making Large-Scale SVM Learning Practical, chapter 11.MIT-Press.

Jung, T., Makeig, S., Humphries, C., Lee, T., Mckeown, M., Iragui, V., Sejnowski, T. (2000) Removing Electroencephalographic Artifacts by Blind Source Separation. Psychophysiology, 37(2):163-17

Jung, T.P., Makeig, S., Stensmo, M., and Sejnowski, T.J., 1997. Estimating Alertness from the EEG Power Spectrum. IEEE Transactions on Biomedical Engineering, 4(1):60-69, January

Pleydell-Pearce, C.W., Whitecross, S.E., and Dickson, B.T.. 2003. Multivariate Analysis of EEG: Predicting Cognition on the basis of Frequency Decomposition, Inter-electrode Correlation, Coherence, Cross Phase and Cross Power. In Proceedings of 38th HICCS

Schmidt, F. and Thews, G. (editors) (1997). Physiologie des Menschen. Springer

Smith, M., Gevins, A., Brown, H., Karnik, A., and Du, R., 2001. Monitoring Task Loading with Multivariate EEG Measures during Complex Forms of HumanComputer Interaction. Human Factors, 43(3):366-380.

Tsochantaridis, I., Hofmann, T., Joachims, T., and Altun, Y. (2004). Support Vector Machine Learning for Interdependent and Structured Output Spaces. In Proceedings of the ICML.

Vesanto, J., Himberg, J., Alhoniemi, E., and Parhankangas, J. (2000). SOM Toolbox for Matlab 5 . Technical report, Helsinki University of Technology.

Zschocke, S. (1995). Klinische Elektroenzephalographie. Springer 